\title{
An Improved Genetic Algorithm Application in a Multi-Objective Design of a Benchmark Cogeneration System
}

\author{
Abdorreza Alavi Gharahbagh, Meisam Babaie, and Davoud Abbasi
}

\begin{abstract}
Multi-Objective Optimization of a benchmark cogeneration problem known as CGAM cogeneration system has been carried out from Exergetic, Economic and Environmental aspects simultaneously. One of the most suitable optimization techniques, known as GA, has been developed here. For using this approach a hybrid fitness function is defined and used to find the optimal solutions with respect to the aforementioned objective functions. This Multi-Objective GA with normalized hybrid fitness function has been considered to decrease the computational cost.

CGAM Problem designs a cogeneration plant which delivers $30 \mathrm{MW}$ of electricity and $14 \mathrm{~kg} / \mathrm{s}$ of saturated steam at $20 \mathrm{bars}$. The thermodynamic modeling has been implemented comprehensively while economic analysis of this system conducted. Consideration of Five decision variables in modeling process made the final optimal solution more realistic in comparison with previous studies in this field. Finally the result of optimization is compared with conventional and multi objective Particle swarm optimization method and the advantages of proposed method are shown.
\end{abstract}

Index Terms - Multivariable systems; CGAM cogeneration system; Genetic Algorithm; Multi-Objective Particle Swarm Optimizer (MOPSO)

\section{INTRODUCTION}

John Holland's pioneering book Adaptation in Natural and Artificial Systems showed how the evolutionary process can be applied to solve a wide variety of problems using a highly parallel technique that is now called the Genetic Algorithm (GA). The genetic algorithm transforms a population of individual objects, each with an associated fitness value, into a new generation of the population. The genetic algorithm attempts to find an optimum (or best) solution to the problem by genetically breeding the population of individuals over a series of generations.

The two important parameters in GA methods are accuracy and rate of convergence. A good GA method needs low iteration (fast convergence) for getting accurate answer.

A major problem in using GA is how to choose the Probability of mutation $\left(\mathrm{P}_{\text {mute }}\right)$. The uncertain nature of this

Manuscript received April 1, 2011

Abdorreza Alavi Gharahbagh is with the department of electrical and computer engineering, Islamic azad university, shahrood branch, Po box 36155/163, shahrood, IRAN. (e-mail: R_alavi@iau_shahrood.ac.ir).

Meisam Babaie is with the department of mechanical engineering, K.N Toosi University of Technology, Tehran, Iran. (e-mail: meisam.babaie@yahoo.com).

Davoud Abbasi is with Islamic azad university, shahrood branch, shahrood, IRAN. (e-mail: davoudabbasi.iau@gmail.com). parameter is a disadvantage. There is a critical unknown value for $\mathrm{P}_{\text {mute }}$. Choosing $\mathrm{P}_{\text {mute }}$ above the critical value lead to high and also constant accuracy and the number of iterations increase, while smaller $P_{\text {mute }}$ values result low accuracy and fewer iterations. There are few values for this parameter that guarantee answer accuracy with low iteration. In order to soften this problem we use the modified mutation method in this work [1]. The modified mutation method has two major advantages in compare with simple mutation; first $P_{\text {mute }}$ in this method is replaced with two new parameters $\left(\mathrm{P}_{\text {mute }}\right.$ and De $_{\text {rate }}$ ) which alleviate the problem of $P_{\text {mute }}$ tuning. Second the answer accuracy in this algorithm is high but not constant similar to simple mutation methods.

Nowadays we face with many systems that have more than one objective and should be optimized from different aspects. GA is a very suitable method for optimizing these complex systems. With increasing demand for energy in different areas, in recent design of modern energy systems that have more than one objective, the importance of applying a reliable and fast algorithm is clear. One of the mot famous and applicable systems in the field of power generation is "cogeneration". It is the production of electrical energy and useful thermal energy from the same energy source. Cogeneration is important for numerous reasons. The first is that capturing the waste heat from power generation can result in an increase in efficiency [4]. This offers significant potential savings in energy costs. Additionally cogeneration is also more environmentally friendly than conventional fossil fuel power plants [5]. Optimization of thermal systems is one of the most important subjects in the energy engineering field [6]. Among the thermal systems, combined cycle cogeneration systems are analyzed by advanced thermodynamic topics. These topics include exergy, thermoeconomics and environment. Exergy analysis, which is the combination of first law and second law of thermodynamics, helps to highlight the thermodynamic inefficiencies of a system. It is clear that improving a system thermodynamically without considering economics and environment is misleading. Hence in design of thermal systems an integrated procedure should be performed to consider all these aspects. Many researchers have started to develop links between exergy and economics. As a result, a new area called thermoeconomics or exergoeconomics has been formed [4]. The aim of the thermoeconomic analysis is to calculate the cost of each product of the systems and investigate the cost formation process in the systems. An example for comparison of different thermoeconomic methodologies to design optimization of a cogeneration plant 
has been presented as a test case, known as the CGAM problem [5-9]. These works initially represent a paradigmatic application of a single optimization problem. Pioneer designers utilized mathematical approaches in their optimization in which these methods suffer from the difficulty to find the final global optimum. Moreover they considered economic aspects only and as it is clear, single objective optimization in thermal systems is not excellent. We can not only consider the cost objectives and forget other aspects. In many cases system performance and environmental impacts are as important as cost effects and for small increase in cost term; more effective solutions will be reached. In multi-objective optimization problems mathematical approaches are unsuccessful in finding the global optimum and most of them terminate at local optimum. Application of multi-objective optimization method in thermal systems is not very old. In 2002, Toffolo et al [10], considered two-objectives: energetic and economic objectives in optimization of CGAM problem. In their work shortcoming of the conventional mathematical optimization approaches in finding global optimum was relatively maintained; they used evolutionary algorithms (MOEAs) with a MATLAB Simulink model and presented a Pareto optimum frontier instead of single optimum solution of the conventional single objective optimization. They improved their work by adding the environmental impact and introduced a three objectives optimization problem [11]. Unit damage costs were devoted to NOx and $\mathrm{CO} 2$ emissions and environmental objective was introduced in cost term. However their work still suffers some shortcoming arose from simplification in selecting of decision variables and constraints. To reduce the number of non-feasible solutions that their optimization algorithm may be faced during the optimization procedure, variable $\varepsilon_{a p}$ was preferred to exit temperature on air side of the Air Preheater $\mathrm{T}_{3}$ (variable used in the original CGAM problem). Furthermore among the five decision variables in original CGAM problem, they chose three of them $\left(r_{c p}, T_{4}, \varepsilon_{a p}\right)$ while other two were held constant. In 2008 Sayyaadi [12], used a more suitable method in economic modeling (TRR method). He added environmental objective with cost objective function and introduced a Thermoenvironomic objective function and utilized this objective with exergetic aspect in two multi-objective optimization approaches. In comparison with previous studies in this field ([10,11, and 12]), this work utilizes a faster and more simple algorithm in optimization procedure (GA) without any simplification with all five decisions variables. GA algorithm can overcome the problem of non feasible solution which has been faced in previous studies. No decision variables are changed or fixed and all variables and constraints are in accordance with the original CGAM problem. These improvements lead to results which are more realistic than corresponding results obtained before. Only [2] that used MOPSO has these properties and the result of this is look like GA. With applying MOPSO, as it has been done in [2], a Pareto optimal solution is introduced. But in this method the Pareto optimal solution is not introduced and final optimal solution is obtained directly. The advantage of our method is its simplicity and flexibility in compare with

\section{MOPSO.}

\section{GENETIC AlgORITHM}

It is common when working with design of energy systems to have situations with more than one objective. For instance, the objectives can simultaneously be to minimize the negative environmental impact of the process, maximize the profit and to maximize the safety of the process. These problems are referred to as multi objective Mathematical programming problems. Equation 1 shows how a multi objective optimization problem can be formulated mathematically:

$$
\min f_{j}(X) \forall j \in\{1,2,3, \ldots . k\} \text { subject to } X \in L
$$

Where we have $k \geq 2$ objective functions.

GA is a methodology in evolutionary computation that is commonly used for these problems.

The genetic algorithm transforms a population of individual objects, each with an associated fitness value, into a new generation of the population. It is based on Darwinian principle of reproduction and survival of naturally occurring genetic operations such as crossover and mutation. The genetic algorithm attempts to find an optimum (or best) solution to the problem by genetically breeding the population of individuals over a series of generations. It is very simple to implement and solves problems very quickly.

In this work we develop a Multi-Objective GA with a normalized fitness function to decrease the computational cost dealing with some multi-objective optimization test problems taken from literature.

\section{A. General methods}

We can divide GA methods by three main operators: selection, crossover and mutation.

\section{1) Selection}

a) Selection initial samples

- Randomly create initial samples in search space.

- Create sequential initial samples in search space.

\section{b) Selection scheme in algorithm}

- The probability to choose a certain sample is proportional to its fitness. Algorithm at last is permit to select $\mathrm{N} / 2$ samples from $\mathrm{N}$ initial samples ${ }^{1}$.

- Algorithm chooses N/2 samples with better fitness and discards other samples ${ }^{2}$.

- The probability to choose a certain sample is proportional to its fitness but if the sample with best fitness discards, algorithm replaces this sample with one of selected samples and discards $i^{3}$.

\section{2) Cross over}

- One-point crossover: two strings cut at a randomly chosen position and swapping the two tails. One-point crossover is a simple method for GAs.

\footnotetext{
Roulette wheel

Ideal selection

${ }^{3}$ Elitism
} 
- N-point crossover: Instead of only one, $\mathrm{N}$ breaking points are chosen randomly. Every second section is swapped.

- Segmented crossover: Similar to N-point crossover with the difference that the number of breaking points can vary.

- Uniform crossover: For each position, it is decided randomly if the positions are swapped.

- Shuffle crossover: First a randomly chosen permutation is applied to the two parents and then $\mathrm{N}$-point crossover is applied to the shuffled parents.

\section{3) Mutation}

- Inversion of single bits: With probability $P_{\text {mute }}$, one randomly chosen bit is negated.

- Bitwise inversion: The whole string is inverted bit by bit with probability $P_{\text {mute }}$

- $\quad$ Random selection: With probability $P_{\text {mute }}$, the string is replaced by a randomly chosen one.

Any combination of these operator types makes a GA method. In practice, a desired GA method rapidly and effectively optimizes complex, highly nonlinear, multidimensional systems. A desired GA method should be faster than other methods and more precise.

Among these operators, defining mutation is more crucial than others because of its uncertain nature. Setting this probability higher than critical value, lead to high answer accuracy. The drawback is increasing the numbers of iterations. If this value is assumed smaller than critical value, answer accuracy will be poor and number of iterations will be low. There is a narrow band for this parameter that guarantee answer accuracy with low iteration. Because of certain nature of other parameters in compare with mutation, they are not as important as mutation.

\section{B. Proposed method}

In this method, mutation operator has been changed for improving GA parameters. Mutation only occurs in positions where bit value of all samples at that position is the same [1]. It is obvious that the mutation in proposed method occurs only in defined bits while general methods apply mutation in all bits. This modified mutation point selection lead to better system performance. Assume one point crossover occur in group1 and group2 at defined positions. As can be seen from fig1, after mutation for samples 2 and 4 the bit value in mutation point is negated.

At the next step if child with mutation remain in selection process, the mutation is said to be good and algorithm continues without any change. On the other hand if these children do not remain in selection process, mutation is not appropriate. This indicates increasing the probability of appearing zeroed bit at this location in the final answer. This means that probability that defined bit (at mutation point) be zero at final answer is big.
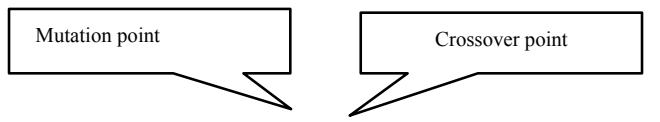
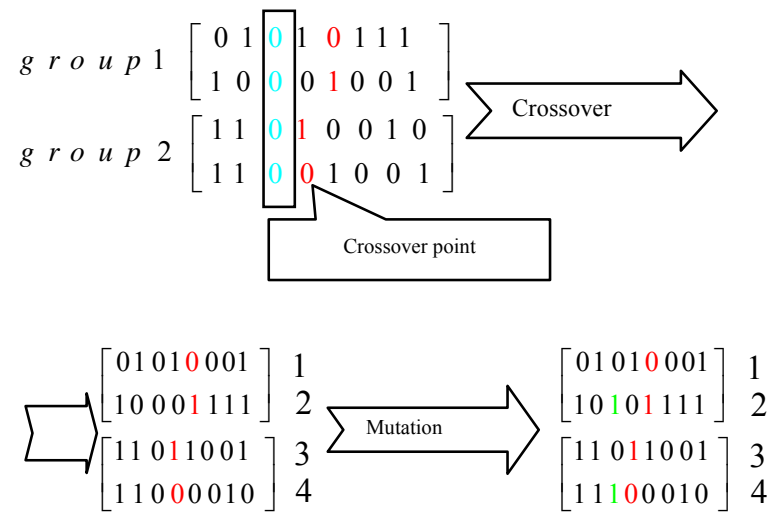

Fig 1: Parents and child in an example of proposed algorithm.

Facing this conditions lead us to finding a way that decrease the probability of mutation in defined bit. In the above example the first assumed $P_{\text {mute }}$ is 0.5 because the mutation occurred in half of child. As a solution we can assume at next step this probability is 0.25 as a result defined bits in one of four children is negated.

In this method we define different probability of mutation for each position (In figl eight separate $P_{\text {mute }}$ ). This probability $P_{\text {mute }}$ is similar for all bits comprising the mutation point.

In order to evaluate the probability changes in a better way we define $P_{\text {Start }}$ instead of $P_{\text {mute }}$. $P_{\text {Start }}$ is the probability of changing bits in mutation point at first mutation which is 0.5 in the above example. $P_{\text {Start }}$ is assumed to be equal in all bit positions. Another major parameter in the proposed method is decrease rate which is defined $D e_{\text {rate }}{ }^{4}$. D $e_{\text {rate }}$ describes the rate of decreasing $P_{\text {Start }}$ between two successive mutation steps. In above example $P_{\text {start }}$ is assumed 0.5 and 0.25 for first and second steps, respectively. $D e_{\text {rate }}$ is defined by division of these two probabilities which is 0.5 . Following this procedure, the third $P_{\text {mute }}$ computed for above example is 0.125 (Fig 2).

$$
\begin{aligned}
& P_{\text {start }}=0.5 \rightarrow P_{\text {mute } 1}=P_{\text {start }} \times D e_{\text {rate }}=0.25 \rightarrow \\
& P_{\text {mute } 2}=P_{\text {mute } 1} \times D e_{\text {rate }}=0.125
\end{aligned}
$$

Fig 2: Probability changes in proposed method and effect of $D e_{\text {rate }}$

It is obvious that $D e_{\text {rate }}$ value would be bigger than 1 and should be positive. Setting this parameter close to 1 may result in low convergence rate (very high number of iterations). Setting this parameter to a big value leads to a general mutation system with low $P_{\text {mute }}$.

The main parameters of the GA set as follows:

- Initial samples are created randomly in search space.

- Only N/2 samples with better fitness are chosen and other samples are discarded (Ideal selection).

\footnotetext{
${ }^{4}$ Decrease Rate
} 
- Algorithm uses N-point crossover: Instead of only one, $\mathrm{N}$ breaking points are chosen randomly. Every second section is swapped.

- Mutation method is chosen Proposed method such as [1]

In this study, the number of initial samples is assumed to be 4096 , the number of cross point $=7$, the number of bits in each variable is selected as 10 . The parameters $\mathrm{P}_{\text {Start }}=0.5$ and $D e_{\text {rate }}=1.2$ are applied to guarantee answer accuracy. These values present a reasonable global search characteristic of the optimization process.

\section{Application of Algorithm to a Case Study}

In 1990, a group of concerned specialists in the field of Thermoeconomics (C. Frangopoulos, G. Tsatsaronis, A. Valero, and M. von Spakovsky) decided to compare their methodologies by solving a predefined and simple problem of optimization: the CGAM problem, which was named after the first initials of the participating investigators [5]. The CGAM problem originally is an economic optimization of a simple cogeneration system which involves physical, thermodynamic, and economic models. It assumes ideal gas behavior and constant heat capacities. The CGAM Problem designs a cogeneration plant which delivers $30 \mathrm{MW}$ of electricity and $14 \mathrm{~kg} / \mathrm{s}$ of saturated steam at 20 bars. The installation consists of an air compressor (AC), air preheater $(\mathrm{APH})$, combustion chamber (CC), gas turbine (GT), and HRSG. Air preheater uses thermal energy from the combustion gas leaving gas turbine to heat the air entering the combustion chamber. Structure of this cogeneration plant is shown in fig 1. HRSG is composed of an economizer (EC) section where the feed water is heated and an evaporator (EV) section where the heated water is vaporized into steam. Other specifications and operating condition of the CGAM problem for base case design are [4]:

$\mathrm{T} 1=298.15 \mathrm{~K}, \mathrm{P} 1=1.01325 \mathrm{bar} ; \mathrm{T} 8=298.15 \mathrm{~K}, \mathrm{P} 8=20 \mathrm{bar}$; $\mathrm{T} 10=298.15 \mathrm{~K}, \mathrm{P} 10=12 \mathrm{bar}$;

$\mathrm{T} 3=850 \mathrm{~K} ; \mathrm{T} 4=1520 \mathrm{~K} ; \mathrm{P} 2 / \mathrm{P} 1=10 ; \eta \mathrm{sc}=0.86 ; \eta \mathrm{st}=0.86$

\section{THERMODYNAMIC MODEL}

Thermodynamic modeling has been carried out in accordance to the procedure presented in [5, 10 and 11]. Utilized thermodynamic model is developed based on the following basic assumptions [5, 10 and 11]:

- All processes are steady state.

- Principle of ideal-gas mixture is applied for air and combustion products.

- Fuel is natural gas and it is assumed to be $100 \%$ methane. Methane is an ideal gas.

- Heat loss from the combustion chamber is considered to be $2 \%$ of the fuel lower heating value. All other components are considered adiabatic.

- Constant pressure loss ratios are considered in components.

- Restricted dead state is $\mathrm{P} 0=1.013$ bar and $\mathrm{T} 0=25^{\circ} \mathrm{C}$.

- $\quad 3 \%$ and $5 \%$ pressure losses are assumed for air and gases in the air preheater, respectively.
- $\quad 5 \%$ pressure losses are assumed for gases in HRSG and combustion chamber.

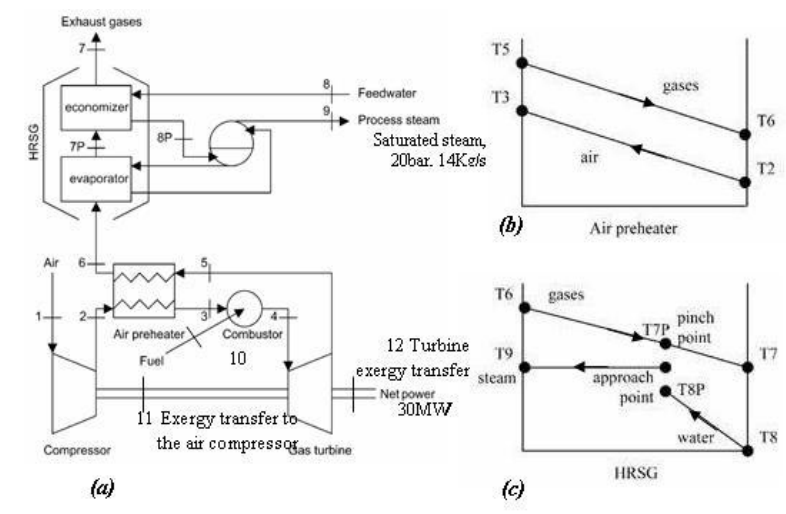

Fig 3: Schematic flow diagram of the CGAM [4].

\section{THERMO ECONOMIC MODEL}

To keep the conformity with other studies in this field, we used the simple economic modeling [2, 5, 10 and 11]. The economic model takes into account the cost of components, including amortization and maintenance, and the cost of fuel consumption. In order to define a cost function which depends on the optimization parameters of interest, component costs have to be expressed as functions of thermodynamic variables [4]. In the CGAM problem, purchase cost functions for each plant component are already supplied. In this research, these equations with their related constants have been considered in accordance with $[2,5,10$ and 11$]$.

Governing equation of thermoeconomic model for the cost balancing of a component of an energy system is as follow [4]:

$$
\sum_{j=1}^{n}\left(c_{j} \dot{E}_{j}\right)_{k, \text { in }}+\dot{Z}_{k}^{C I}+\dot{Z}_{k}^{\text {OM }}=\sum_{j=1}^{m}\left(c_{j} \dot{E}_{j}\right)_{k, \text { out }}
$$

Where $\mathrm{cj}$ is the unit cost of exergy for the jth stream to/from the component, $\dot{E}_{j}$ is exergy flow for the jth stream to/from the component and $\dot{Z}_{k}^{C I}$ and $\dot{Z}_{k}^{\text {OM }}$ are related cost of capital investment and operating and maintenance for the component $\mathrm{k}_{\mathrm{th}}$ obtained in economic model. In equation $5, \mathrm{n}$ and $m$ are total number of inlet and outlet exergy to/from the component $\mathrm{k}_{\mathrm{th}}$, respectively. Developing equation 5 for each component of CGAM problem along with auxiliary costing equations (according to $\mathrm{P}$ and $\mathrm{F}$ rules, see [4]) leads to the following system of equations. The system of 12 equations and 12 unknowns as indicated by equation 6 is solved to obtain the cost of streams 1 to 12 for CGAM problem.

\section{COMBustion Pollutants}

The original CGAM problem does not perform calculations on formation of pollutants within the combustion chamber. A simple model, based on semi-analytical correlations [13], is added here to determine pollutant emissions to setup of an environmental objective function. Adiabatic flame temperature in the primary zone of the combustion chamber is derived from the expression by Gulder [14]:

$$
T_{p z}=A \sigma^{\alpha} \exp \left(\beta(\sigma+\lambda)^{2}\right) \pi^{x} \theta^{y} \psi^{z}
$$


The adiabatic flame temperature is used in the semi-analytical correlations proposed by Rizk and Mongia $[13,14]$ to determine the pollutant emissions in grams per kilogram of fuel:

$$
\begin{aligned}
& N O_{x}=\frac{0.15 E 16 \tau^{0.5} \exp \left(-71100 / T_{p z}\right)}{p_{3}^{0.05}\left(\Delta p_{3} / p_{3}\right)^{0.5}} \\
& C O=\frac{0.179 E 9 \exp \left(7800 / T_{p z}\right)}{p_{3}^{2} \tau\left(\Delta p_{3} / p_{3}\right)^{0.5}}
\end{aligned}
$$

$$
\left[\begin{array}{cccccccccccc}
1 & 0 & 0 & 0 & 0 & 0 & 0 & 0 & 0 & 0 & 0 & 0 \\
1 & -1 & 0 & 0 & 0 & 0 & 0 & 0 & 0 & 0 & 1 & 0 \\
0 & 1 & -1 & 0 & 1 & -1 & 0 & 0 & 0 & 0 & 0 & 0 \\
0 & 0 & 0 & 0 & \frac{1}{\dot{E}_{5}} & \frac{-1}{\dot{E}_{6}} & 0 & 0 & 0 & 0 & 0 & 0 \\
0 & 0 & 1 & -1 & 0 & 0 & 0 & 0 & 0 & 1 & 0 & 0 \\
0 & 0 & 0 & 0 & 0 & 0 & 0 & 0 & 0 & 1 & 0 & 0 \\
0 & 0 & 0 & 1 & -1 & 0 & 0 & 0 & 0 & 0 & -1 & -1 \\
0 & 0 & 0 & \frac{1}{\dot{E}_{4}} & \frac{-1}{\dot{E}_{5}} & 0 & 0 & 0 & 0 & 0 & 0 & 0 \\
0 & 0 & 0 & 0 & 0 & 0 & 0 & 0 & 0 & 0 & \frac{1}{\dot{E}_{11}} & \frac{-1}{\dot{E}_{12}} \\
0 & 0 & 0 & 0 & 0 & 1 & -1 & 1 & -1 & 0 & 0 & 0 \\
0 & 0 & 0 & 0 & 0 & \frac{1}{\dot{E}_{6}} & \frac{-1}{\dot{E}_{7}} & 0 & 0 & 0 & 0 & 0 \\
0 & 0 & 0 & 0 & 0 & 0 & 0 & 1 & 0 & 0 & 0 & 0
\end{array}\right]\left[\begin{array}{c}
\dot{C}_{1} \\
\dot{C}_{2} \\
\dot{C}_{3} \\
\dot{C}_{4} \\
\dot{C}_{5} \\
\dot{C}_{6} \\
\dot{C}_{7} \\
\dot{C}_{8} \\
\dot{C}_{9} \\
\dot{C}_{10} \\
\dot{C}_{11} \\
\dot{C}_{12}
\end{array}\right]=\left[\begin{array}{c}
0 \\
\dot{Z}_{A C} \\
\dot{Z}_{A P H} \\
0 \\
\dot{Z}_{C C} \\
F C_{L} \\
\frac{F}{C_{G T}} \\
-\dot{Z}_{G T} \\
0 \\
0 \\
\dot{Z}_{H R S G} \\
0 \\
0
\end{array}\right]
$$

\section{OBJECTIVE FunCtions, DECISION VARIABLES AND CONSTRAINTS}

Three objective functions of the multi-criteria optimization problem are the total exergetic efficiency (to be maximized), the total cost rate of products (to be minimized) and the environmental impact (to be minimized). Third objective function expresses the environmental impact as total pollution damage cost $(\$ / \mathrm{s})$ due to $\mathrm{CO} 2$ and NOx emissions by multiplying their respective flow rates by their corresponding unit damage cost [3] $\left(C_{\mathrm{CO}_{2}}\right.$ and $C_{\mathrm{NO}_{x}}$ are equal to $0.02086 \$ / \mathrm{kgCO} 2$ and $6.853 \$ / \mathrm{kgNOx}$, respectively [11]). Mathematical formulation of objective functions is as following,

Exergetic:

$$
\varepsilon_{\text {tot }}=\frac{\dot{W}_{N E T}+\dot{m}_{\text {steam }}\left(e_{9}-e_{8}\right)}{\dot{m}_{\text {fuel }} e_{\text {fuel }}}
$$

Cost:

$$
\dot{C}_{P, t o t}=\dot{C}_{F, t o t}+\dot{Z}_{t o t}^{C I}+\dot{Z}_{t o t}^{O M}
$$

Environmental:

$$
\dot{C}_{e n v}=C_{\mathrm{CO}_{2}} \dot{m}_{\mathrm{CO}_{2}}+c_{\mathrm{NO}_{X}} \dot{m}_{\mathrm{NO}_{X}}
$$

In this paper, for keeping the consistency with the original CGAM problem [4-9], same decision variables have been selected as follows. Decision variables are:

- Compressor pressure ratio $\mathrm{p} 2 / \mathrm{p} 1$

- Isentropic efficiency of the compressor $\eta s c$

- Isentropic efficiency of the turbine $\eta \mathrm{st}$

- Temperature of the air entering the combustion chamber T3

- Temperature of the combustion products entering the gas turbine $\mathrm{T} 4$
Although the decision variables may be varied in optimization procedure, each decision variable is normally required to be within a given range as follow:

$$
\begin{aligned}
& 6 \leq p_{2} / p_{1} \leq 16 \\
& 0.6 \leq \eta_{s c} \leq 0.9 \\
& 0.6 \leq \eta_{s t} \leq 0.92 \\
& 700 \leq T_{3} \leq 1000 \mathrm{~K} \\
& 1200 \leq T_{4} \leq 1550 \mathrm{~K}
\end{aligned}
$$

Heat exchange between hot and cold streams in air preheater and HRSG should satisfy the following feasibility constraints:

Air preheater:

$$
\begin{aligned}
& \mathrm{T} 5>\mathrm{T} 3 \\
& \mathrm{~T} 6>\mathrm{T} 2
\end{aligned}
$$

\section{HRSG:}

$$
\begin{aligned}
& \Delta \mathrm{TP}=\mathrm{T} 7 \mathrm{P}-\mathrm{T} 9>0 \\
& \mathrm{~T} 6 \geq \mathrm{T} 9+\Delta \mathrm{TP} \\
& \mathrm{T} 7 \geq \mathrm{T} 8+\Delta \mathrm{TP} \\
& \mathrm{T} 7 \mathrm{P}>\mathrm{T} 8 \mathrm{P} \\
& \mathrm{T} 7 \geq 378.15 \mathrm{~K}
\end{aligned}
$$

Last constraint is an additional constraint with respect to the original CGAM problem imposed on exhaust gases temperature, which must not fall below $378.15 \mathrm{~K}\left(105^{\circ} \mathrm{C}\right)$. This limitation is considered to prevent the condensation of water vapor exist in the combustion products at outlet section of economizer. The condensation of water vapor in presence of carbon dioxide may leads to formation of carbonic acid which is corrosive material and can damage the economizer surface.

\section{RESULTS AND DISCUSSION}

In order to evaluate advantages and robustness of the GA method the results are compared to the original CGAM problem [5] solved using conventional mathematical optimization approach and MOPSO approach [2]. In this regard, the thermodynamic and economic model is built based on simple thermodynamic and economic models utilized in [5, 9]. As we mentioned before, in original CGAM problem the economic objective had been considered alone without considering the environmental or efficiency aspects and an optimum point from the sight of economics was introduced. In table 1 the comparison between original CGAM problem and a point on Pareto domain which has the least cost in [2] and GA is shown:

As it can be seen from table below, the point introduced from GA algorithm is totally more effective than conventional method, while the cost was increased a bit; exergetic efficiency and environmental effects are improved. This point is approximately better than MOPSO point computed in [2]. The most advantage of our method is its independent nature of Pareto domain. In other words all conditions should be summarized in fitness function. With applying this method we could find a good solution very fast and simple with considering all objectives and without any need for introducing the Pareto domain. In the other word, other advantage of this algorithm is its speed and simplicity. 
TABLE I: COMPARISON OF RESULTS FOR OPTIMIZATION OF ORIGINAL CGAM PROBLEM OBTAINED IN THIS PAPER WITH THOSE OBTAINED BY CONVENTIONAL OPTIMIZATION APPROACHES IN [5] AND MOPSO IN [2]

\begin{tabular}{|c|c|c|c|}
\hline $\begin{array}{c}\text { Objective function, } \\
\text { decision variables, } \\
\text { costing and operating } \\
\text { parameters, etc }\end{array}$ & $\begin{array}{c}\text { Conventional } \\
\text { optimization } \\
\text { approaches } \\
\text { presented in [5] }\end{array}$ & $\begin{array}{c}\text { Optimization via } \\
\text { MOPSO [2] the } \\
\text { least cost point } \\
\text { among pareto } \\
\text { domain }\end{array}$ & $\begin{array}{c}\text { Optimization via } \\
\text { Gresented in } \\
\text { this work }\end{array}$ \\
\hline $\begin{array}{c}\text { Total Cost Rate (\$/s) } \\
\text { Environmental Cost } \\
\text { Rate (\$/s) }\end{array}$ & 0.362009 & 0.36379691 & 0.3809 \\
\hline $\begin{array}{c}\text { Exergetic efficiency } \\
(\%)\end{array}$ & 50.664 & 51.26109 & 52.56 \\
\hline$T_{3}(K)$ & 914.28 & 920.19 & 844.6 \\
\hline$T_{4}(K)$ & 1492.63 & 1492.47 & 1496.6 \\
\hline$\eta_{s c}$ & 0.8468 & 0.8306 & 0.8695 \\
\hline$\eta_{s t}$ & 0.8786 & 0.8456 & 0.9006 \\
\hline$p_{2} / p_{1}$ & 8.52 & 7.7 & 12.0802 \\
\hline
\end{tabular}

\section{CONCLUSIONS}

An alternative to previously presented calculus based optimization approaches named GA algorithm was utilized for multi-objective optimization of typical cogeneration system called CGAM problem. The proposed evolutionary algorithm was shown to be a powerful and effective tool in finding the optimal solution for the choice of optimum design variables in the CGAM cogeneration plant in comparison to the conventional mathematical optimization algorithm and MOPSO method. In the proposed method, instead of introducing Pareto domain such as MOPSO, normalized hybrid fitness is used. The proposed method has two main advantages in compare other methods. First advantage of presented method was obtained from the comprehensive thermodynamic and economic modeling with no need for simplification and change of decision variables or constraints that was implemented along with the GA algorithm which was shown that is able to achieve better result than conventional mathematical approach and other evolutionary algorithm. The second is its simplicity and flexibility in compare MOPSO method. It was shown that multi-criteria optimization approach, which is a general form of single objective optimization, enables us to consider various and ever competitive objectives.

\section{REFERENCES}

[1] Alavi gharahbagh. Abdorreza, and Abolghasemi. vahid. "A Novel Accurate Genetic Algorithm for Multivariable Systems", World Applied Sciences Journal 5 (2), pp. 137-142, 2008.

[2] Meisam Babaie, Hoseyn Sayyaadi, Mohammad Reza Farmani, Alireza Novinzadeh. "Exergy, Cost and Environment as objectives In Particle Swarm Optimization of a benchmark cogeneration system", proceeding of ESDA 2010, Istanbul, Turkey, July 12-14, 2010.

[3] Lefebvre AH. "Gas Turbine Combustion. Ann Arbor (MI)", Edwards Brothers, 1998.

[4] Bejan A, Tsatsaronis G, And Moran M. "Thermal and design optimization", New York: John Wiley and Sons Inc, 1996.

[5] Valero A, Lozano MA, Serra L, Tsatsaronis G, Pisa J, Frangopoulos CA, von Spakovsky MR. "CGAM problem: definition and conventional solution”, Energy 1994, 19(3):279-86.

[6] Tsatsaronis G, Pisa J. "Exergoeconomic evaluation and optimization of energy systems: application to the CGAM problem", Energy 1994, 19(3):287-321.

[7] Frangopoulos CA. "Application of thermoeconomic optimization methods to the CGAM problem”, Energy, 1994, 19(3):323-42.

[8] Von Spakovsky MR. Application of engineering functional analysis and optimization of the CGAM problem. Energy 1994, 19(3):343-64.

[9] Valero A, Serra L, Lozano MA, Torres C. "Application of the exergetic cost theory to the CGAM problem", Energy 1994, 19(3):365-81.

[10] Toffolo A, Lazzaretto A. "Evolutionary algorithms for multi-objective energetic and economic optimization in thermal system design", Energy 2002, 27:549-67.

[11] Toffolo A, Lazzaretto A. "Energy, economy and environment as objectives in multi-criteria optimization of thermal system design", Energy 2004, 29: 1139-1157.

[12] Sayyaadi H. "Multi-objective approach in thermoenvironomic optimization of a benchmark cogeneration system", Applied Energy, Volume 86, Issue 6, June 2009, Pages 867-879

[13] Rizk NK, Mongia HC. "Semianalytical correlations for NOx, CO and UHC emissions", Journal of Engineering for Gas Turbine and Power 1993, 115(3):612-9.

[14] Gülder Ö. L. "Flame temperature estimation of conventional and future jet fuels", Journal of Engineering for Gas Turbine and Power 1986, 108(2):376-80. 